\title{
Computer-aided study of the gas flow within a natural gas storage and the associated collection network
}

\author{
Alina Gligor ${ }^{1, *}$, Cristian Deac ${ }^{1}$, and Marius Bibu $^{1}$ \\ ${ }^{1}$ Lucian Blaga University of Sibiu, Faculty of Engineering, 4 E. Cioran st., 550025 Sibiu, Romania
}

\begin{abstract}
The current paper presents a computer-aided analysis of the way in which a natural gas storage created in a depleted gas deposit and the associated gas collection network are operated, both during the extraction phase and during the injection phase. In a first step, an analysis is carried out on a two-dimensional, nonstationary model for the gas storage, based on the deposit's geometry and the rock properties, but also on the gas motions. Also, a dynamic simulation of the injection and extraction processes is unfolded, allowing the study and optimisation of elements such as well placement and gas flows through the wells with the goal of maximising the storable gas quantities. A second model is then generated for the gas collection network associated with the natural gas storage, using the simulation software Simone. Its main benefit is allowing the simulation and modelling of nonstationary flows through the network. The two models are closely interconnected, in that for the extraction stage, the output from the first model is used as input for modelling the collection network, while for the injection phase, the results from modelling the collection network are used as entry data for the gas storage model.
\end{abstract}

\section{Introduction}

Natural gas storages are facilities built with the purpose of holding, during the warm season, the largest possible amount of gases which can then be used for heating or other purposes during the cold season [1]. The processes of injecting gas into a storage space and then extracting gas from that storage are complex and involve the consumption of relatively large amounts of energy.

Gas storages realised in depleted gas deposits are complex structures that can occupy wide areas and are operated by means of several gas wells. An efficient management of these storages involves the constant monitoring of the processes taking place and an advanced knowledge of the gas pillow's spatial distribution in the storage space.

While a review of the relevant speciality literature has revealed several studies that concern the modelling and optimisation of processes unfolding in gas deposits or reservoirs [1-4], most of these refer to either general problems or to the specific problem of storing carbon dioxide in depleted gas deposits [3 - 4]. Furthermore, it should be mentioned that no specific studies have targeted the reservoir in the area described by this paper; also, processes unfolding in the gas collecting network are usually treated separately.

* Corresponding author: alina.gligor@ulbsibiu.ro 
The current paper describes the manner of linking two numerical models that allow the modelling of an injection process: on the one hand a numerical nonstationary two-dimensional model for the simulation of storage-extraction processes in time and space that allows the obtaining of a dynamic spatial image of the gas distribution in the storage and on the other hand a model for the gas collecting network, created with the help of the professional simulation software Simone allowing to model the transit of a non-stationary gas flow through the collection network.

\section{The model of the gas collecting network}

The first step in realising the combined model was to generate the model of the gas collecting network leading to the gas deposit using the numerical simulator Simone, developed by the SIMONE Research Group s.r.o. from the Czech Republic; this software uses as inputs the network configuration as well as the storage process dynamics.

In order to allow the validation of its results, the authors have chosen to allow the model's input data to reflect the reality on the ground, the pipe dimensions, for example, being based on GIS data from an actual gas storage located in Romania. Thus, the studied collection network includes on the one hand adduction pipes that link the gas wells to the collection points (groups) and on the other hand adduction pipes that link the collecting pipes to the gas delivery/reception points on the National (Gas) Transportation System (NTS). Specifically, the analysed network consists of a compressor station, 5 well groups, 8 collecting pipes, 19 adduction pipes and 19 wells.

Figure 1 presents the flow diagram of the studied collection network for the gas deposit.

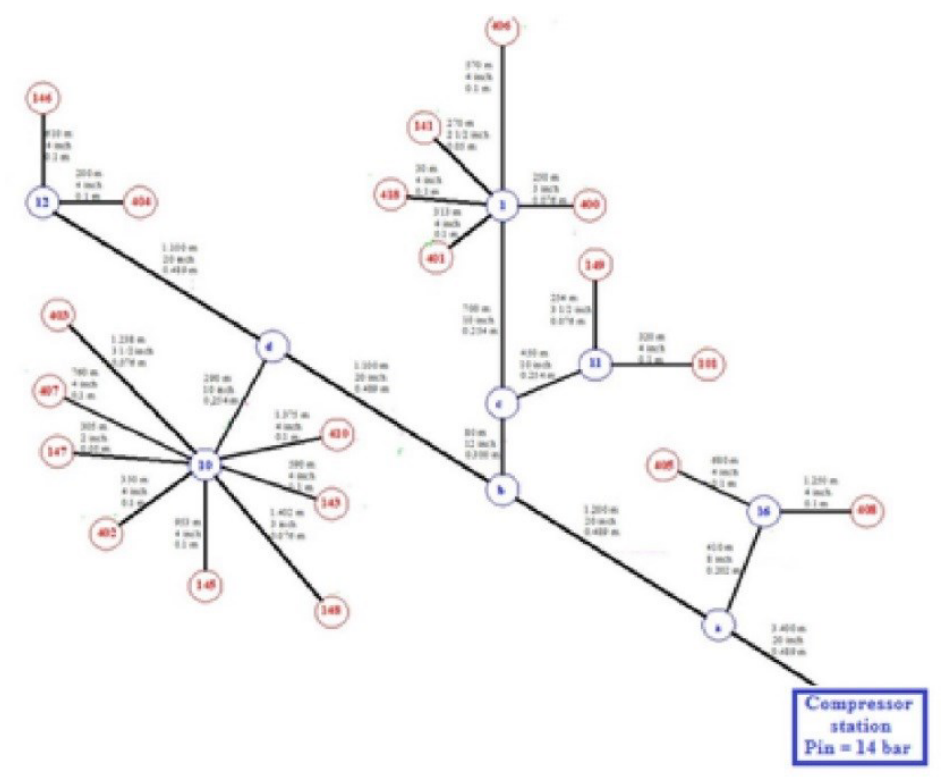

Fig. 1. The diagram of collection pipes in the studied network

In order for the model to be realistic, the working scenarios defined in the program needed to contain some constraints due to the unsteady gas flow in the national transportation system. An important constraint that arose was the limited transport capacity of the collecting network. The flow rates injected/extracted into/from the gas storage must not exceed the transport capacity of the collecting network.

The compressor station is designed to raise the pressure of the gas taken from NTS pipes to a level that allows it to be introduced into the gas storage. At the points where the gas leaves the 
collection system and enters the well, regulators allow the defining of injection parameters. These parameters depend on the well's configuration (diameter, length) and on the depleted deposit's properties (permeability, porosity, deposit pressure around the well).

Based on the created gas collecting network model, the authors created in a next step dynamic simulation scenarios for the gas injection. Here, the parameters such as gas flows and pressures injected daily into the storage were used as boundary conditions for the storage model; the model allowed to calculate for each considered day the spatial distribution of the injected gas volumes, the total amount of gases injected up to that day as well as the pressure field in the storage.

The total duration of the considered gas injection stage was of 132 days, but due to the large amount of data needed to be fed into the scenario and due to the calculation possibilities offered by the available software and hardware, this time span was divided on scenarios with a duration of 10 days each (except for the last scenario that lasts only 2 days), the next scenario starting from the parameters resulting at the end of the simulation of the previous scenario. The full injection cycle resulted from the totality of the 14 scenarios for that process.

\section{The Numerical Model of the Gas Storage}

The numerical model of the gas storage can be used to assess the gas amounts that should be injected and to accept these amounts into the storage or, to the contrary, to reject the amounts that would surpass the holding possibilities of the storage.

For the construction of this model too, the authors have started from the real geometry of the considered gas storage. Since it can be considered that the upper and lower limits of the storage, respectively, have a relatively flat profile and that the thickness is largely constant, a twodimensional model has been chosen, reflecting only the extent on a horizontal plane.

As stated, the numeric model was designed to reflect the storage's real geometry. Therefore, the model's integration field had to be identical with the shape of the storage. This has been achieved by defining a so-called informatic field map with the purpose of allowing the running of calculations for the case of irregularly shaped integration fields.

The integration process is done at the same time with reading the field map. With the help of the information that is stored here, calculations can be carried out according to the type of points: some of the points require integration calculations, others require the definition of boundary limit conditions or limit conditions on wells, respectively etc. The modelling of processes occurring in the studied gas storage uses flow equations for a flat storage, defined in Cartesian coordinates [2, 3]:

$$
\frac{\partial}{\partial x}\left(\frac{k h}{\mu z} \frac{\partial^{2} p^{2}}{\partial x}\right)+\frac{\partial}{\partial y}\left(\frac{k h}{\mu z} \frac{\partial p^{2}}{\partial y}\right)+M_{s} h R T=2 m h \frac{\partial}{\partial \tau}\left(\frac{p}{z}\right)
$$

where: $\mathrm{x}, \mathrm{y}, \mathrm{z}$ - spatial coordinates; $\mathrm{p}$ - pressure; $\mathrm{k}$ - permeability; $\mathrm{h}$ - height of the storage; $\mu$ - dynamic viscosity of the gas; Ms - mass flow of the source; R - gas constant; $\mathrm{T}$ - temperature; $\mathrm{m}$ - porosity;

For the analysed storage in the depleted gas deposit, the chosen method was a Du FortFrankel integration with two temporary steps, because it offers unconditional stability and a high processing speed. The employed interactive scheme is the one indicated in equation 2:

$$
\begin{aligned}
& u_{i j}^{n+1}=\frac{2 \alpha_{x}}{1+2 \alpha_{x}+2 \alpha_{y}}\left(u_{i+1, j}^{n}+u_{i-1, j}^{n}\right)+\frac{2 \alpha_{y}}{1+2 \alpha_{x}+2 \alpha_{y}}\left(u_{i, j+1}^{n}+u_{i, j-1}^{n}\right)+ \\
& +\frac{1-2 \alpha_{x}-2 \alpha_{y}}{1+2 \alpha_{x}+2 \alpha_{y}} u_{i j}^{n-1}+2 B M_{s}
\end{aligned}
$$


The integration process requires the defining of initial conditions and of limit conditions. These allow in turn to define various scenarios applied to the gas storage model [5]. Therefore, the difference between the gas injection stage simulated on the model and the simulated extraction stage is made by the specific limit conditions.

The process of injecting gas into the deposit starts from an initial gas pressure (and amount) in the storage, called a "gas pillow" (Fig. 2). It can be assumed that the pressure is initially distributed evenly throughout the storage.

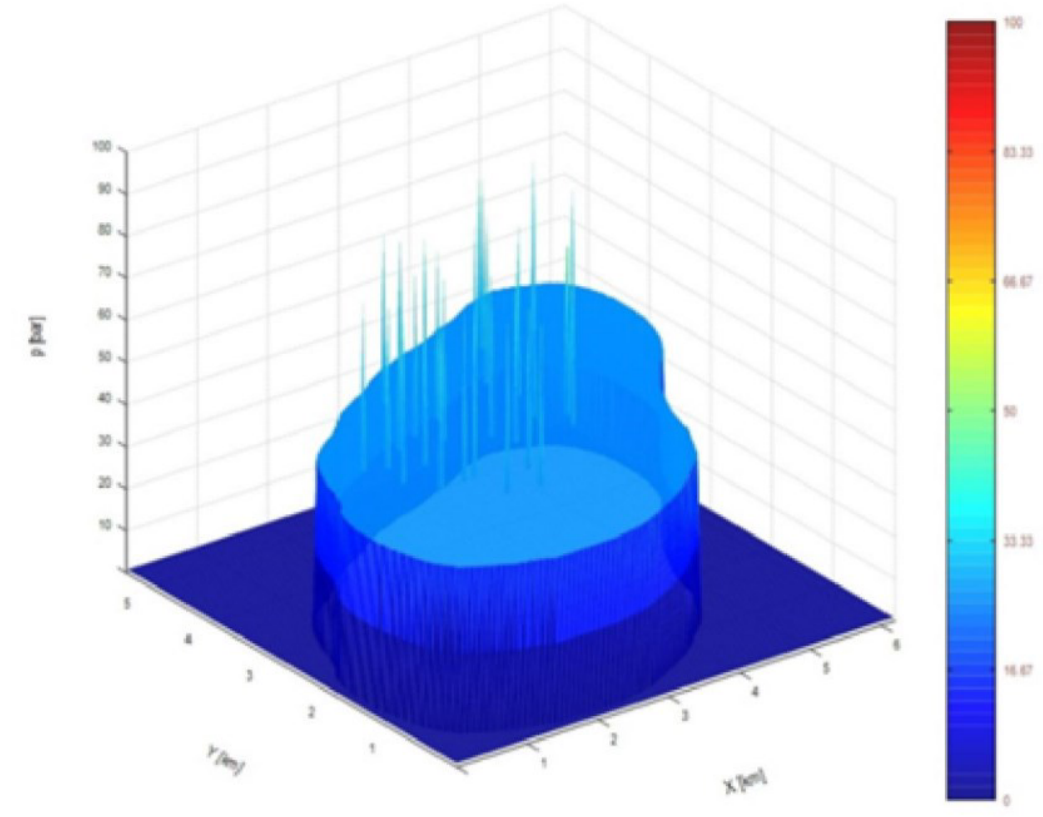

Fig. 2. Gas pillow and storage wells

The initial conditions are determined at the beginning of the integration process. Since the flow equation is defined based on pressure, the initial conditions for this model can be written as:

$$
p_{i j}^{0}=p_{i, \min }\left\{\begin{array}{l}
i=1,2 \ldots n x \\
j=1,2 \ldots n y
\end{array}\right\}
$$

Limit conditions are used to define the manner in which the integration process unfolds. In this case, the authors have employed, two types of limit conditions: boundary limit conditions and limit conditions on wells.

Boundary limit conditions are determined in the boundary points of the integration field (points that limit the area of the considered gas storage). Since for gas storages, the boundaries have to be considered impermeable, the speed of the gas in the boundary points is zero. Considering a flat (two-dimensional) storage area, there are only two sets of boundary conditions: one for the gas speed on the direction of the Ox axis and the other for the gas speed on the direction of the Oy axis (Equations 4 and 5).[3]

$$
v_{x}=-\frac{k}{\mu} \frac{\partial p}{\partial x} \cong-\frac{k}{\mu} \frac{\left(P_{i, j}^{n+1}\right)_{f}-P_{i+1, j}^{n+1}}{\Delta x}=0
$$




$$
\mathrm{v}_{\mathrm{y}}=-\frac{\mathrm{k}}{\mu} \frac{\partial \mathrm{p}}{\partial \mathrm{y}} \cong-\frac{\mathrm{k}}{\mu} \frac{\left(\mathrm{P}_{\mathrm{i}, \mathrm{j}}^{\mathrm{n}+1}\right)_{\mathrm{f}}-\mathrm{P}_{\mathrm{i}+1, \mathrm{j}}^{\mathrm{n}+1}}{\Delta \mathrm{y}}=0
$$

where:

$\mathrm{x}, \mathrm{y}$ - coordinates of the points within the storage area;

$\mathrm{p}$ - pressure;

$\mathrm{k}$ - permeability;

$\mu$ - dynamic viscosity.

Using the relations (4) and (5), the boundary conditions have been rewritten as follows: $\left(P_{i, j}^{n+1}\right)_{f}=P_{i \pm 1, j}^{n+1}$ on the Ox direction $\left(P_{i, j}^{n+1}\right)_{f}=P_{i, j \pm 1}^{n+1}$ on the Oy direction

where the index f refers to boundary, i.e. $\left(P_{i, j}^{n+1}\right)_{f}$ represents the pressure of the point with the coordinates $i, j$ located on the boundary of the domain.

For the injection stage, the limit conditions in the gas wells describe the injected flow moving through the well. The flow is defined by the well dimensions, as well as by the pressure and permeability of the storage-containing layer. The flow then allows the generation, within the model, of the injection and extraction pressures that provides the actual transportation of that flow through the gas well.

In order to inject a mass flow of gas $\dot{m}$ into a well located at the coordinates $(i, j)$, the existence of a pressure $\left(P_{i, j}^{n+1}\right)$ in that well. This value can be determined from the limit conditions, based on the law of mass balance. This leads to the expression of $\left(P_{i, j}^{n+1}\right)$ given in equation 6:

$$
P_{i, j}^{n+1}=\frac{\frac{m}{\frac{k}{\mu}}+\left(P_{i+1, j}^{n} \rho_{x_{1}}+P_{i-1, j}^{n} \rho_{x_{2}}\right) d y+\left(P_{i, j+1}^{n} \rho_{y_{1}}+P_{i, j-1}^{n} \rho_{y_{2}}\right)}{\left(\rho_{x_{1}}+\rho_{x_{2}}\right) d y+\left(\rho_{y_{1}}+\rho_{y_{2}}\right) d x}
$$

For the extraction stage, the limit condition would not change, but the flow m extracted from the well would need to be introduced with the minus sign.

In the case of a gas storage in the settlement stage, that is, gases are neither not injected nor extracted, the pressure in the storage tends to homogenize itself.

The limit condition for this case is then given by the sum of the average pressures from nearby points:

$$
P_{i j}^{n+1}=\frac{\left(p_{i+1, j}^{n}+P_{i-1, j}^{n}+P_{i, j+1}^{n}+P_{i, j-1}^{n}\right)}{4}
$$

\section{Results of the simulation for the stage of gas injecting stage}

\subsection{Results for the simulation on the gas collecting network model}

After running the simulations with the model defined as presented above, there have been obtained several results that, after comparison with the data from the real gas storage, allowed the validation of the conceived model and confirmed its accuracy. In the following there are presented the most relevant ones results. mainly as graphs in order to be more suggestive.

Figure 3 presents the variation of flows through the surface system, after 20 days of injection. 


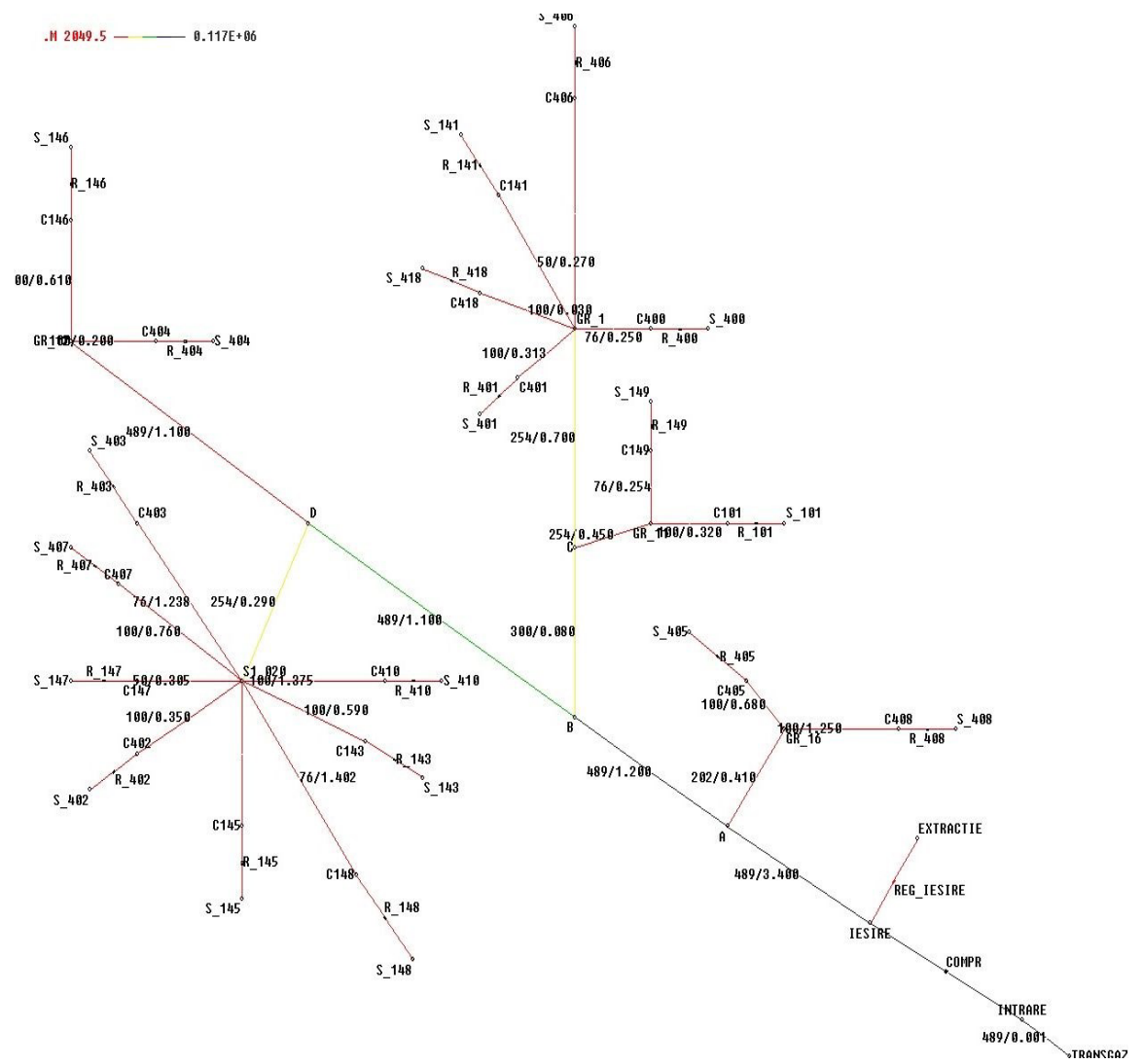

Fig. 3. Variation of the gas flows in the collecting network after 20 days

In order to synthetise the results of all simulation scenarios considered, there were created graphs of the main parameters for the whole injection period. Thus, figure 4 presents the variation of the gas compression pressure. It can be seen that in this case, the compression pressure is approximately constant.

Figure 5 presents the variation of the total gas flow injected into the gas storage. Beneath the daily variations, there can also be noticed a descending trend - as the injection period increases, the gas amount entering the storage is smaller, because the pressure in the deposit increases.

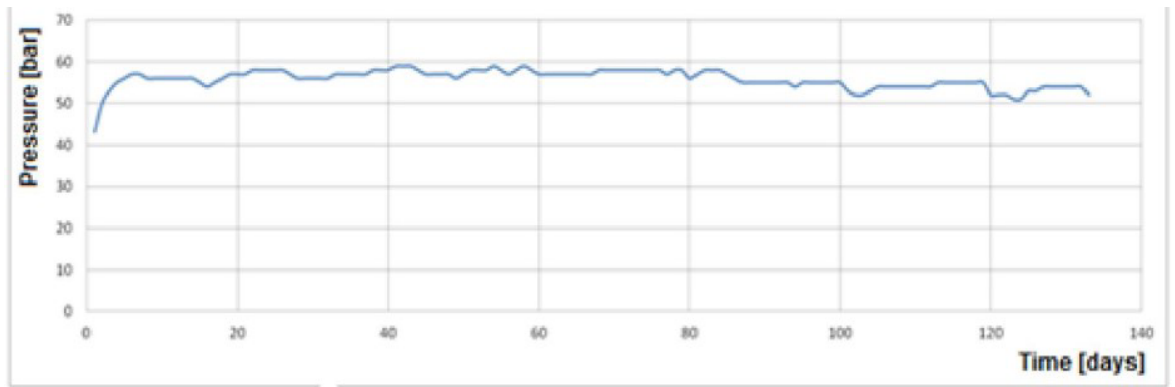

Fig. 4. Variation of the gas compression pressure during the injection stage 


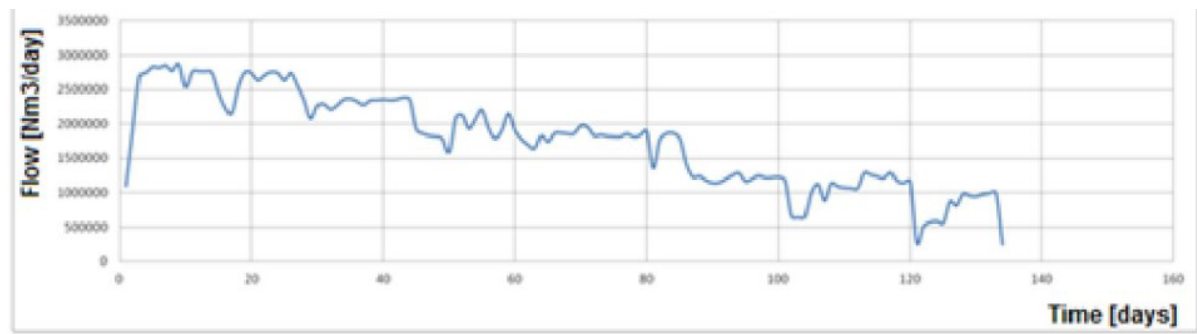

Fig. 5. Variation of the injected gas flow

The total flow injected into the deposit is divided on each well. Figure 6 presents the variation of flows on 5 of the existing wells.

Even if the general descending trend is similar to the one in figure 5, when analysing each well in part, it can be noticed that the injected flow differs very much from day to day. This needs to be correlated with the pressure variation in the wells, a variation exemplified in figure 7 for other 5 wells.

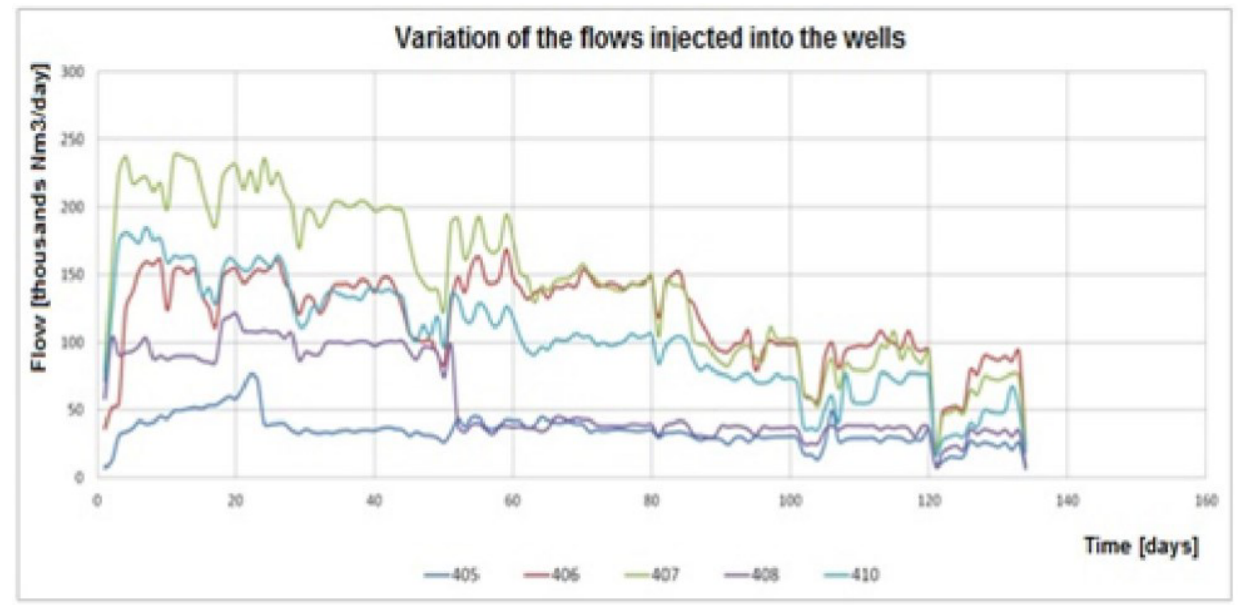

Fig. 6. Variation of the flow injected into the wells

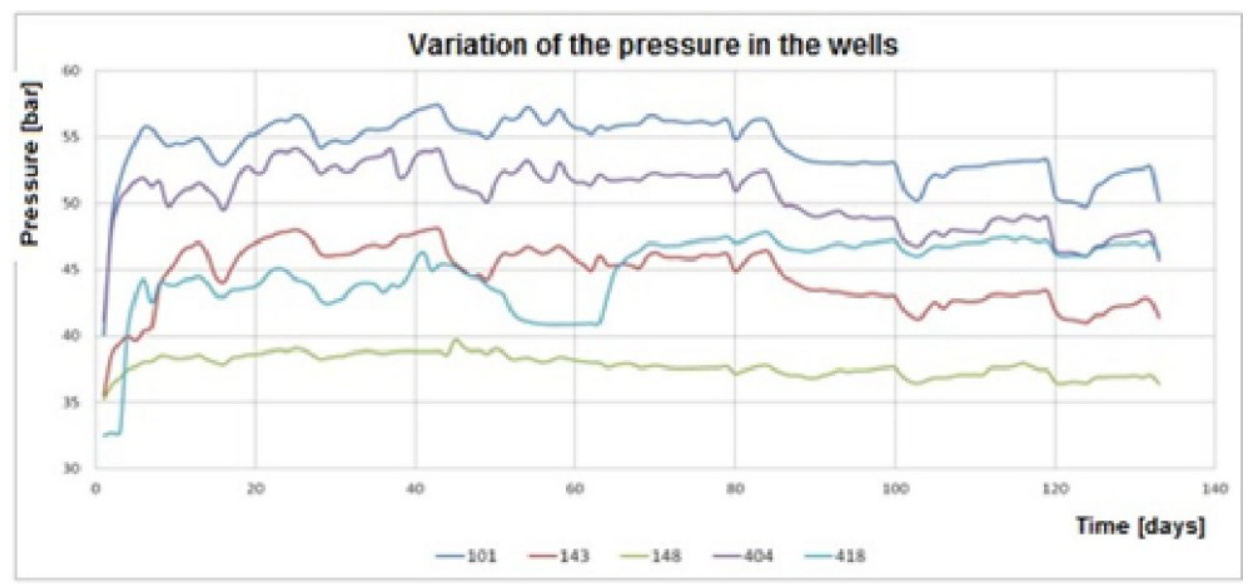

Fig. 7. Variation of the pressure in the wells 


\subsection{Results for the simulation on the gas storage model}

Using the storage model for each day of injection, the flows and pressures obtained from the simulation of the transport network of the storage were imposed as limit conditions for the wells on which the injection is done. Using these conditions, a complex simulation was realised for the entire duration of the injection process.

Figure 8 presents the average daily pressures and the cumulative values for the entire period, for all 19 wells considered. The unevenness is due to the variation of the injected daily flows. This graph also presents the average pressure in the deposit (yellow curve) and the total amount of injected gases (blue curve). The slope of this blue curve depends on the daily flows that are injected, so if in the last part of the injection process (after 90 days) the slope decreases, it is because the daily flows that are injected are also smaller.

Figure 9 presents the spatial distribution of the pressure at the end of the injection stage. The peaks on the surface correspond to the pressures in the wells. However, it can be also noticed that at the end of the considered injection period, due to the current location of the injection wells, there remains a large part of the original deposit volume which is not filled by the stored gases (green area). This can be corrected by placing new wells in the areas that show a deficit in stored gas.

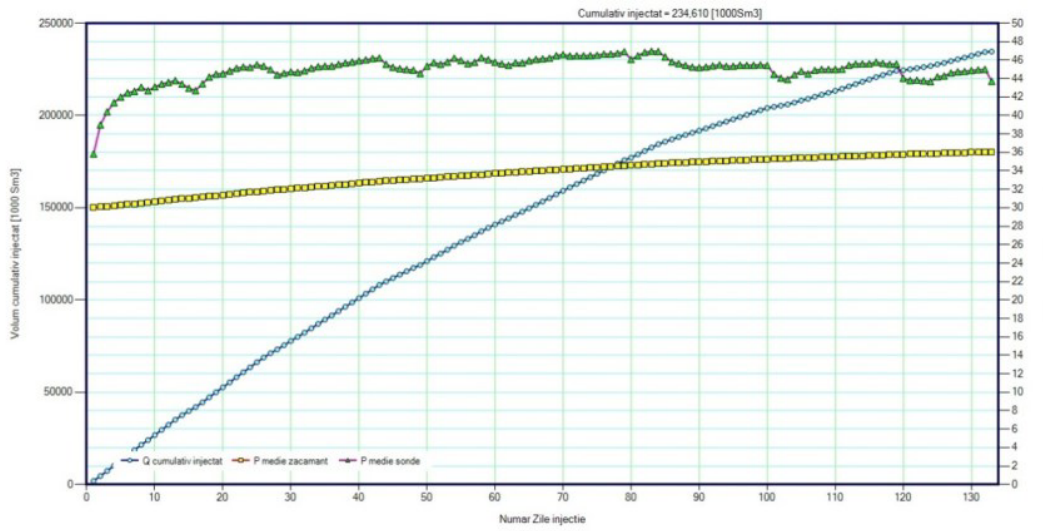

Fig. 8. Average and total values for the injection process

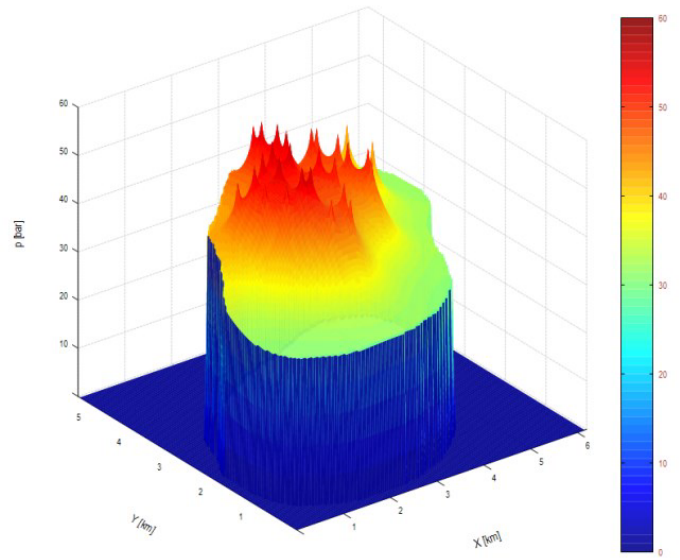

Fig. 9. Pressure values throughout the storage area at the end of the injection cycle 
After injection there follows a settlement stage during which pressures homogenize themselves. In Fig. 10 the pressures in the storage are represented at the end of the settlement stage of 14 days. One can notice the homogenization of pressures in the field of injection wells, but that here too, the storage is not filled evenly with gases even if during the settlement stage a part of the gases has migrated towards the boundary of the storage.

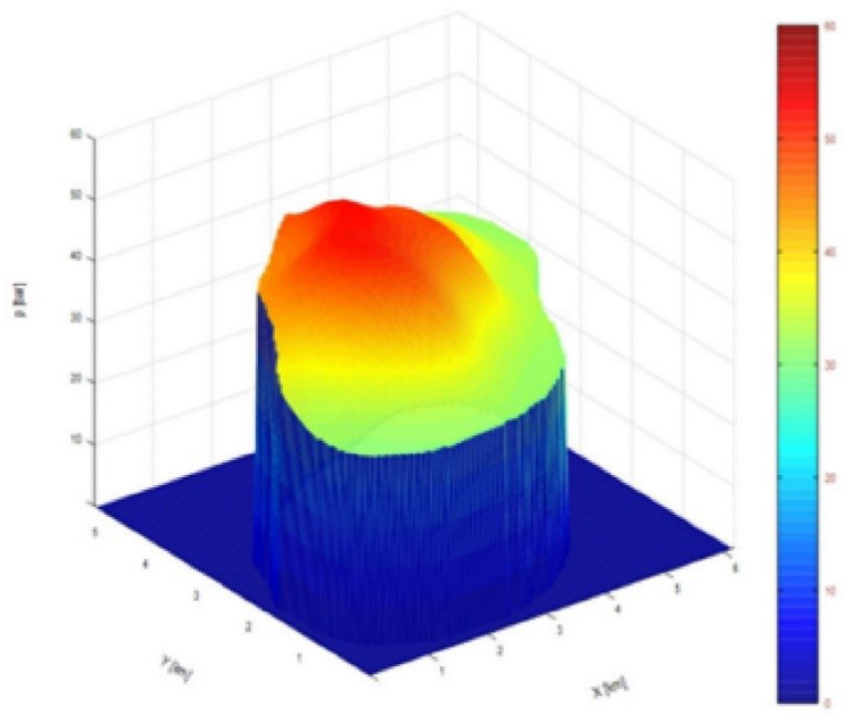

Fig. 10. Pressure values throughout the storage area at the end of the settlement stage after injection

\section{Conclusions}

As has been presented in this paper, the developed numerical simulation allowed to define the settings required for the daily usage of operating elements such as controllers, valves, compression station etc. that facilitate the transportation of the gas through the gas collection network from the compression station to the well for the injection phase and reverse for the extraction phase under optimal pressure conditions.

By using a dynamic model of the collecting network for the field/ deposit, it was possible to simulate full injection / extraction cycles in real conditions.

The model of both the collection network and the gas storage is especially useful for monitoring their behaviour, which is carried out by comparing the acquired SCADA information with the expected values. If larger differences between individual data points are detected, or if data evolution trends emerge that differ from the projected ones, corrections can be determined following quick simulation processes.

The developed model can simulate also the period between two cycles during which gases are neither injected nor extracted (settlement period).

For the gas storage, the developed model determines the values of parameters such as pressure, gas saturations, gas volumes for any given point of the storage and for any moment during the analysed period. For example, the pressure matrix can be determined precisely and can be represented as a 3D graph.

The numerical model described here also facilitates operations such as the placement of new wells (extraction points) in order to achieve a more homogeneous distribution of the pressures within the storage. 


\section{References}

1. M. Albulescu, S. Neacsu, C. Eparu, S. Bambo, S. Suditu, Termotehnica, XV, S1 (2011)

2. D. He, A. Jia, G. Ji, Y. Wei, H. Tang, Petroleum Exploration and Development, 40, 1 (2013)

3. I. Crețu, Modelarea numerica a miscarii fluidelor in medii poroase (Technical Publishing House, Bucharest, 1980)

4. C. Eparu, R. Radulescu, D. Stoica, Annals of the Ovidius University, Chemistry series, 24, 2 (2013)

5. C. Eparu, S. Suditu, A. Prundurel, Annals of the Ovidius University, Chemistry series, 24, 2 (2013)

6. S. Neacsu, C. Eparu, Journal of Eastern Europe Research in Business and Economics, 2013 (2013)

7. S. Neacsu, Comprimarea si lichefierea gazelor (Romconvert Publishing House, Ploiesti, 2002)

8. S. Neacsu, C. Eparu, Proceedings of the 21 st International Business Information Management Association (IBIMA) Conference, Vienna, Austria (2013)

9. S. Neacsu et al., Environmental Engineering and Management Journal, 9, 10 (2010)

10. S. Neacsu, S. Suditu, C. Popescu, Proceedings of the 1st International Conference on Power and Energy Systems, Chania, Greece (2013)

11. S. Neacsu, S. Suditu, D. Stoica, Annals of the Ovidius University, Chemistry Series, 24, 2 (2013)

12. M. Nikolaou, Computers and Chemical Engineering 51 (2013).

13. H. Zangeneh, S. Jamshidi, M. Soltanieh, International Journal of Greenhouse Gas Control, 17 (2013)

14. *** Simone 5.7, http://www.simone.eu/simone-company-about.asp, accessed 21.02.2017 